\title{
Severe Fever with Thrombocytopenia Syndrome, Japan, 2013-2017
}

\author{
Yusuke Kobayashi, ${ }^{1}$ Hirofumi Kato, Takuya Yamagishi, Tomoe Shimada, Tamano Matsui, \\ Tomoki Yoshikawa, Takeshi Kurosu, Masayuki Shimojima, Shigeru Morikawa, ${ }^{2}$ Hideki Hasegawa, \\ Masayuki Saijo, Kazunori Oishi, ${ }^{3}$ SFTS Epidemiological Research Group Japan ${ }^{4}$
}

\begin{abstract}
We conducted an epidemiologic study of severe fever with thrombocytopenia syndrome (SFTS) in Japan during 2013-2017. Of 303 cases reported during that period, $133(44 \%)$ were included in this study. The median time between onset of illness and diagnosis of SFTS shortened, from 11.5 to 3.0 days, but the case-fatality rate remained high, at $27 \%$. In 64 patients (48\%), a close contact with companion animals was reported within 2 weeks of disease onset. Of these 64 patients, 40 were surveyed further, and we confirmed that 3 had direct contact with body fluids of ill companion animals; 2 had direct contact with the saliva of an ill feral cat or pet dog. These patients reported no history of tick bite, suggesting that ill companion animals might be a source of SFTS virus transmission. Direct contact with the body fluids of ill companion animals should be avoided.
\end{abstract}

Severe fever with thrombocytopenia syndrome S(SFTS) is an emerging tickborne infectious disease, identified in 2009 in the rural areas of Hubei and Henan provinces in China $(1,2)$; a total of 7,419 cases were reported from 23 provinces during 20102016 (3). SFTS is endemic not only to China but also to South Korea and Japan $(4,5)$. Huaiyangshan banyangvirus (formerly SFTS virus [SFTSV]), the causative agent of SFTS, belongs to the genus Banyangvirus in the family Phenuiviridae. Although the name of the virus has recently been changed from SFTSV to Huaiyangshan banyangvirus by the International Committee on Taxonomy of Viruses (6), the term SFTSV is still used. SFTSV is found in tick species such as Haemaphysalis longicornis, Amblyomma testudinarium, and Ixodes nipponesis in China, South Korea, and Japan (7-9). Antibodies to SFTSV were detected in wild and domestic animals, such as goats, deer, cattle, dogs, and cats, in SFTS-endemic areas of these 3 countries (10-15). SFTSV is thought to circulate in an

Author affiliation: National Institute of Infectious Diseases,

Tokyo, Japan

DOI: https://doi.org/10.3201/eid2604.191011 enzootic environment and to have a tick-vertebratetick cycle (12). In addition, human-to-human transmission through blood and respiratory secretions has been reported from China and South Korea (16-18).

Since the first case of SFTS was reported in Japan in 2013 (5), ad hoc retrospective and prospective surveillance has been conducted by Japan's Ministry of Health, Labor, and Welfare. A total of 23 suspected SFTS cases were retrospectively reported from 2005, of which 11 were confirmed through this surveillance $(5,19)$. SFTS was included in Japan's Infectious Diseases Control Law as a category IV notifiable disease on March 4, 2013. Our previous report describing the nationwide epidemiology of SFTS during April 2013September 2014 demonstrated that the case-fatality rate (CFR) in 49 patients was as high as $31 \%$ (20). As of October 2017, a total of 303 confirmed cases had been reported to Japan's National Epidemiologic Surveillance of Infectious Disease (NESID) (21).

The annual numbers of SFTS patients reported each year during 2013-2017 were 40, 61, 60, 59, and 83, respectively. These patients were reported from 23 of the 47 prefectures in Japan, and the geographic distribution of the SFTS cases expanded gradually each year from western to central Japan (Appendix Figure 1, https:/ / wwwnc.cdc.gov/EID/article/26/4/19-1011-App1. pdf). Most patients had SFTS onset during April-October (Appendix Figure 2), and the annual national notification rates of SFTS ranged from 0.03 to 0.06 cases/100,000 person-years over the study period. These findings were similar to those previously reported for 2013 and 2014 (20).

Studies conducted in China, South Korea, and Japan reported that several clinical and laboratory

${ }^{1}$ Current affiliation: Saitama Prefectural Government, Saitama, Japan.

${ }^{2}$ Current affiliation: Okayama University of Science, Ehime, Japan. ${ }^{3}$ Current affiliation: Toyama Institute of Health, Toyama, Japan.

${ }^{4}$ Group members are listed at the end of the article. 
parameters were associated with a fatal outcome of SFTS $(20,22-25)$. Because no effective therapeutic agents for SFTS are currently available $(22,25,26)$, effective and specific treatments for SFTS must be developed. A recent case report demonstrated that a veterinarian who cared for 3 symptomatic cats, 2 of which were pets, was infected with SFTSV (27). On the basis of such information, we conducted questionnaire surveys of SFTS patients to collect information regarding their direct contact with ill companion animals 2 weeks before illness onset. We conducted a retrospective observational study to identify the changes in the epidemiologic findings of SFTS patients over the study period of March 2013-October 2017 to determine the prognostic factors for SFTS and to evaluate the possible risk of direct exposure to ill companion animals possibly infected with SFTSV.

\section{Methods}

\section{National Surveillance of SFTS in Japan}

NESID defines a case of SFTS as illness in a patient with fever or gastrointestinal symptoms, any laboratory findings, including thrombocytopenia $(<10.0$ $\left.\times 10^{4} / \mu \mathrm{L}\right)$, leukopenia $(<4,000$ cells $/ \mu \mathrm{L})$, or elevated liver enzymes, plus laboratory confirmation of SFTSV infection. The confirmatory examinations include detection of the SFTSV genome using reverse transcription PCR (RT-PCR), which was performed at the local public health institute, the National Institute of Infectious Diseases (Tokyo, Japan), or both, and SFTSV-specific antibody testing using an immunofluorescence assay or an indirect immunoperoxidase assay $(13,28,29)$.

\section{Study Design and Data Collection}

We performed a retrospective observational study of SFTS cases reported to NESID during March 2013October 2017. Physicians were asked to participate in this study by completing a questionnaire sent by mail. Demographic data, social history of outdoor activity for 2 weeks before illness onset, clinical symptoms, and laboratory data for SFTS patients were collected through the first questionnaire. The physicians who agreed to participate in this study collected the information about the patients through their medical charts or by telephone interviews with patients or their family members, after obtaining informed consent. We collected clinical information and laboratory data for the acute phase (within 2 weeks after illness onset). We obtained data for patients whose cases were reported during March-September 2014 from a previous study (18); our study added data collected during October 2014-October 2017. We extracted basic demographic data for each SFTS patient reported during the study period from the NESID database.

We sent a second questionnaire to the physicians who responded to the first questionnaire; the second questionnaire requested information about whether the confirmed SFTS patients had contact with companion animals (such as cats and dogs). The information requested included whether the SFTS patient had close contact with an ill companion animal within 2 weeks before illness onset or direct contact with body fluids of an ill companion animal and the outcome for the ill companion animal.

\section{Statistical Analyses}

We used SPSS Statistics 21.0 for Windows (IBM, https://www.ibm.com) for all statistical analyses. We performed trend analysis for different years by using the Spearman rank correlation test and the Jonckheere-Terpstra test. We used the Pearson $\chi^{2}$ test or the Wilcoxon rank-sum test to compare the characteristics of survivors and nonsurvivors. We used logistic regression modeling for multivariable analysis. All $p$ values were 2 -sided, and $p<0.05$ was considered statistically significant.

\section{Ethics Approval}

This study was conducted under approval by the Medical Research Ethics Committee of the National Institute of Infectious Diseases (approval no. 706). All aspects of the study complied with the Helsinki Declaration. Each patient or their proxy provided written informed consent.

\section{Results}

\section{Epidemiologic and Clinical Characteristics of Study Cases}

Of 303 patients reported to NESID, we included 133 $(43.9 \%)$ whose demographic and clinical data were collected through the first questionnaire in this study (Figure 1). Of these 133 patients, 130 (98\%) had SFTS diagnosed by detection of an SFTSV-specific genome using RT-PCR and 3 (2\%) by detection of an SFTSVspecific antibody. We observed no significant difference in sex and age distribution between these 133 patients and the 170 patients for whom no questionnaire was collected. The median ages of the 2 groups were 73 years (interquartile range [IQR] 65-82 years) for the patients with questionnaires and 75 years (IQR 67-82 years) for those without.

Although the median time from illness onset to initial hospital visit was 3 days (IQR 2-5 days) and 


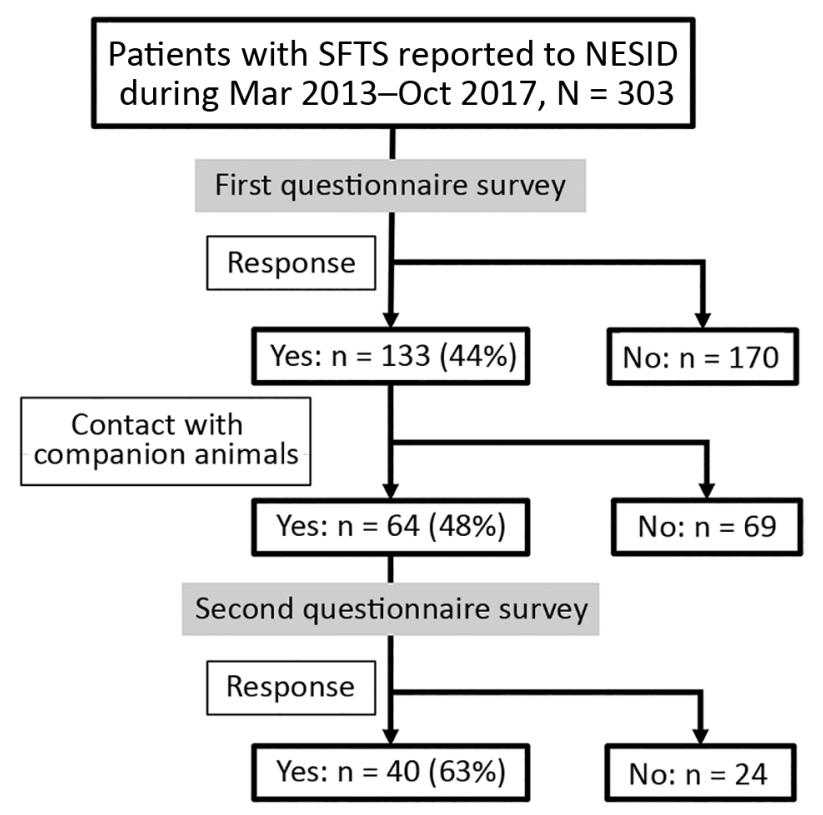

Figure 1. Flow diagram of epidemiologic study of 133 patients with severe fever with thrombocytopenia syndrome, Japan, March 2013-October 2017. NESID, National Epidemiologic Surveillance of Infectious Disease; SFTS, severe fever with thrombocytopenia syndrome.

did not differ significantly between years, the median time between illness onset and a confirmed diagnosis for 133 SFTS cases shortened significantly over the study period $(\mathrm{p}<0.01)$ (Figure 2$)$. The median time between illness onset and a confirmed diagnosis also significantly shortened over the study period for 97 survivors and 36 nonsurvivors $(\mathrm{p}<0.01)$. Thirty-six patients died, corresponding to a CFR of 27\% (Figure

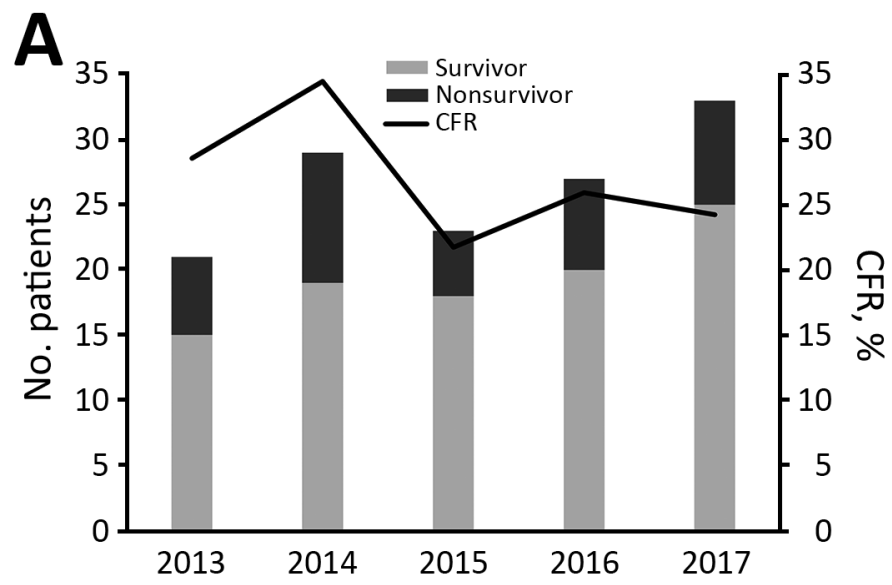

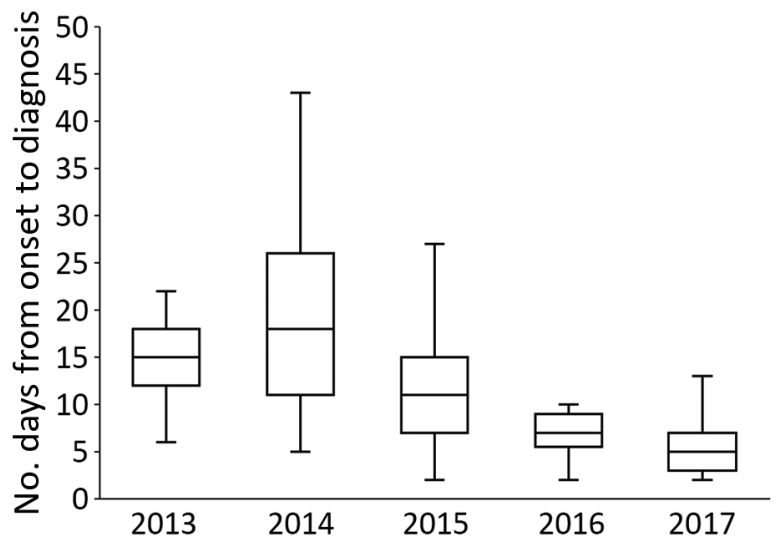

Figure 2. Comparison of time between illness onset and confirmed diagnosis in 133 patients with severe fever with thrombocytopenia syndrome, Japan, March 2013-October 2017. We conducted a trend analysis of time from initial visit to diagnosis over the study period by using the Jonkheere-Trapstra test $(p<0.01)$. In the box plots, the bottom boundary of the box indicates the 25th percentile, the line within the box marks the median, and the top boundary of the box indicates the 75th percentile. Whiskers above and below the box indicate the 10th and 90 th percentiles.

3, panel A). We observed no significant difference in age or sex distribution by year between the 97 survivors and 36 nonsurvivors. The Kaplan-Meier survival curve of 133 SFTS patients demonstrated that most deaths (94\%) occurred within 2 weeks after illness onset (Figure 3, panel B).

Underlying illnesses among these 133 patients included hypertension $(\mathrm{n}=47)$, diabetes mellitus $(\mathrm{n}=27)$, and dyslipidemia $(\mathrm{n}=15)$, as well as a few instances of malignant disease $(n=9)$ (Table 1$)$. The

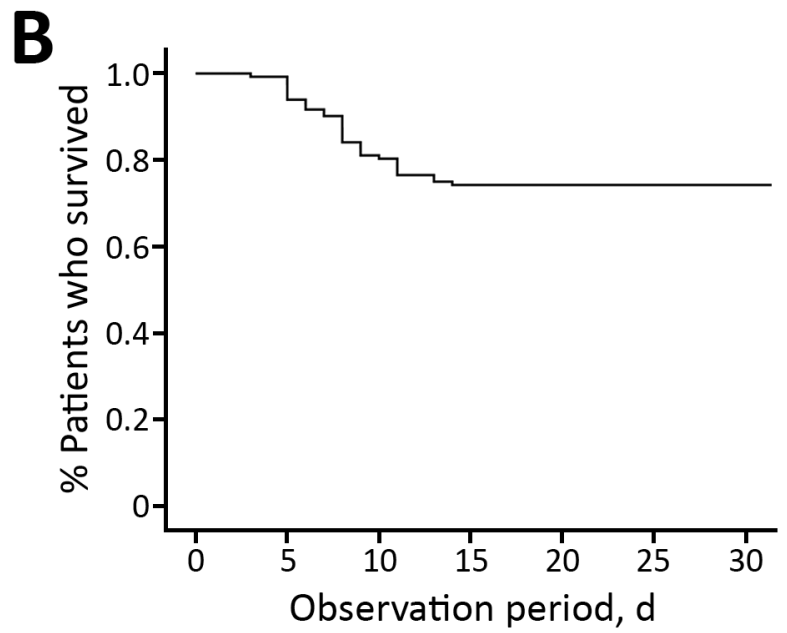

Figure 3. Annual number and CFRs and Kaplan-Meier survival curve of 133 patients with severe fever with thrombocytopenia syndrome, Japan, March 2013-October 2017. A) Trend analysis of CFRs over the study period by using Spearman rank correlation test $(p=0.285)$. B) Kaplan-Meier curve of 133 patients with severe fever with thrombocytopenia syndrome within 30 days after illness onset. CFR, case-fatality rate. 
Table 1. Demographic characteristics of 133 patients with severe fever with thrombocytopenia syndrome, Japan, March 2013October $2017^{*}$

\begin{tabular}{|c|c|c|c|c|}
\hline \multirow[b]{2}{*}{ Characteristic } & \multicolumn{3}{|c|}{ No. (\%) } & \multirow[b]{2}{*}{$\mathrm{p}$ value } \\
\hline & All case-patients, $n=133$ & Nonsurvivors, $n=36$ & Survivors, $\mathrm{n}=97$ & \\
\hline \multicolumn{5}{|l|}{ Sex } \\
\hline M & $63(47)$ & $17(47)$ & $46(47)$ & 0.984 \\
\hline $\mathrm{F}$ & $70(53)$ & $19(53)$ & $51(53)$ & \\
\hline Median age, y (IQR) & $73(65-82)$ & $78(68.25-84.75)$ & $72(63.5-80)$ & $0.015 \ddagger$ \\
\hline \multicolumn{5}{|l|}{ Underlying conditions } \\
\hline Malignant tumor & $9(7)$ & $6(17)$ & $3(3)$ & 0.006 \\
\hline Diabetes mellitus & $27(20)$ & 7 (19) & $12(12)$ & 0.860 \\
\hline Hypertension & 47 (35) & $14(39)$ & $33(34)$ & 0.707 \\
\hline Dyslipidemia & $15(11)$ & $3(8)$ & $12(12)$ & 0.381 \\
\hline None & $36(27)$ & $8(22)$ & $28(29)$ & 0.444 \\
\hline
\end{tabular}

${ }^{*}$ Values are no. (\%) unless indicated. IQR, interquartile range.

†Pearson $\chi^{2}$ test used for all variables except age.

$\ddagger$ Wilcoxon rank-sum test.

proportion of patients with malignant disease was significantly higher among the nonsurvivors (17\%) than among the survivors $(3 \%)(\mathrm{p}<0.05)$. Sixty-four $(48 \%)$ patients were reported to have been bitten by a tick, and 55 (41\%) had traces of a tick bite; 109 $(82 \%)$ had participated in outdoor activities on hills and in fields. Sixty-four (48\%) had close contact with companion animals, such as dogs and cats, within 2 weeks before illness onset.

We reviewed clinical symptoms and laboratory data at initial hospital visit of the 133 patients with available information (Appendix Tables 1, 2). We found no significant difference in neurologic symptoms, except for tremor, between survivors and nonsurvivors. The serum levels of aspartate aminotransferase, alanine aminotransferase, blood urea nitrogen, lactic acid dehydrogenase, and potassium were significantly higher, and activated partial thromboplastin time significantly longer, in nonsurvivors than in survivors.

\section{Prognostic Factors}

To investigate possible prognostic factors at the initial hospital visit, we performed multivariable analysis by selecting factors on the basis of the univariate analysis results, past reports, and clinical importance (Table 2). The multivariable logistic regression analysis indicated that a low platelet count was associated with a fatal outcome (odds ratio [OR] 1.38, 95\% CI 1.07-1.78). The complications of malignant disease (OR 20.83, 95\% CI 1.32-327.70) and presence of tremor at initial hospital visit (OR 17.37, 95\% CI 1.26-239.39) also were associated with an increased risk for death.

\section{Contact with III Companion Animals}

Of 64 patients who responded to the second questionnaire, $40(62.5 \%)$ reported contact with a companion animal within the 2 weeks before illness onset (Figure 1). For 5 of these patients, their companion animal appeared to be ill during this period, and 3 (patients 1 , 2 , and 3) had direct contact with the body fluids of the ill companion animals (Table 3). These three patients had no history of a tick bite. No information was available about the type of body fluid to which patient 1 was exposed. Patient 2 had direct contact with the saliva of a symptomatic feral cat, and she was also bitten by this cat, which subsequently died. No virologic examination of these 2 cats was attempted. Patient 3 had direct contact with the saliva of an ill dog that he owned. RT-PCR detected the presence of SFTSV genome in blood from this dog.

Table 2. Multivariable analysis of prognostic factors for 133 patients with severe fever with thrombocytopenia syndrome, Japan, March 2013-October $2017^{*}$

\begin{tabular}{|c|c|c|c|c|}
\hline \multirow[b]{2}{*}{ Variable } & \multicolumn{2}{|c|}{ Univariable } & \multicolumn{2}{|c|}{ Multivariable } \\
\hline & OR $(95 \% \mathrm{Cl})$ & $p$ value & OR $(95 \% \mathrm{Cl})$ & $p$ value \\
\hline Age & NA & 0.015 & $1.07(0.98-1.16)$ & 0.115 \\
\hline Malignant tumor & $6.13(1.45-26.04)$ & 0.006 & $20.83(1.32-327.70)$ & 0.031 \\
\hline Disorientation & $1.58(0.71-3.52)$ & 0.259 & $1.37(0.30-6.35)$ & 0.687 \\
\hline Tremor & $8.60(1.57-47.04)$ & 0.004 & $17.37(1.26-239.39)$ & 0.033 \\
\hline Platelet & NA & 0.661 & $1.38(1.07-1.78)$ & 0.014 \\
\hline Albumin & NA & 0.791 & $0.99(0.15-6.62)$ & 0.994 \\
\hline ALT & NA & 0.015 & $1.01(1.00-1.02)$ & 0.371 \\
\hline $\mathrm{LDH}$ & NA & 0.012 & $1.00(1.00-1.00)$ & 0.378 \\
\hline CK & NA & 0.591 & $1.00(1.00-1.00)$ & 0.842 \\
\hline APTT & NA & 0.007 & $1.06(0.97-1.16)$ & 0.215 \\
\hline
\end{tabular}


Table 3. Characteristics of 3 patients with severe fever with thrombocytopenia syndrome who had contact with body fluid of an ill companion animal before illness onset, Japan, March 2013-October 2017*

\begin{tabular}{|c|c|c|c|c|c|c|c|c|}
\hline $\begin{array}{l}\text { Patient } \\
\text { no. }\end{array}$ & $\begin{array}{l}\text { Onset } \\
\text { year }\end{array}$ & $\begin{array}{l}\text { Age, } \\
\text { y/sex }\end{array}$ & Outcome & Tick bite & $\begin{array}{c}\text { Species of ill } \\
\text { companion animal }\end{array}$ & $\begin{array}{c}\text { Direct exposure } \\
\text { to ill animal's } \\
\text { body fluid }\end{array}$ & $\begin{array}{l}\text { Outcome of ill } \\
\text { companion } \\
\text { animals }\end{array}$ & $\begin{array}{l}\text { SFTSV detection } \\
\text { from animals }\end{array}$ \\
\hline 1 & 2014 & $46 / F$ & Recovered & No & Cat & Yes & Recovered & Not tested \\
\hline 2 & 2016 & $57 / F$ & Died & No & Cat & Yes (bite) & Died & Not tested $†$ \\
\hline 3 & 2017 & $42 / \mathrm{M}$ & Recovered & No & Dog & Yes & Recovered & Yes \\
\hline
\end{tabular}

\section{Discussion}

In this study, we investigated the epidemiologic and clinical features of 133 SFTS patients identified in Japan during 2013-2017. The overall CFR was 27\%, which did not significantly change over the study period. We found a significant reduction in the interval between illness onset and the SFTS diagnosis and identified that underlying malignant disease, low platelet count, and appearance of tremor at hospital visit were significantly associated with increased risk for death after adjusting for age.

On the basis of national surveillance data, as of October 31, 2017, the proportions of fatal cases during the study period were $35 \%$ in $2013,26 \%$ in $2014,18 \%$ in 2015, 14\% in 2016, and 13\% in 2017 (https:/ / www. niid.go.jp/niid/ja/sfts/sfts-idwrs/7415-sfts-nesid. $\mathrm{html}$ ). Therefore, a discrepancy was noted between the CFRs observed in this study and the proportions of fatal cases in the national surveillance database. We found that the time between illness onset and hospital visit remained unchanged but that the time between illness onset and diagnosis shortened significantly, indicating an increased awareness of this disease among physicians during more recent years in the SFTS-endemic areas of Japan. Because physicians who diagnose a case of SFTS are requested to report the case to NESID immediately after diagnosis, the time between illness onset and reporting had also been shortened (from 14 days [IQR 11-24 days] during 2013-2014 to 6 days [IQR 4-8 days] in 2017).

Our findings demonstrate that deaths commonly occurred within 2 weeks after illness onset (Figure 3, panel B). During the study period, 11 patients ( 1 patient each in 2013 and 2015, 2 patients in 2016, and 7 patients in 2017) died after their diagnosis was reported to NESID. This fact might explain, in part, the discrepancy between the CFRs in this study and the proportions of fatal cases in national SFTS surveillance data.

Our finding that a low platelet count at initial hospital visit was a risk factor for a fatal outcome is in agreement with the results of previous studies $(20,22,23)$. Although age-adjusted underlying malignant disease also was significantly associated with fatal outcome in our study, whether it has a direct effect on the survival of SFTS patients remains uncertain because of the limited number of such cases (n $=9$ ). A previous study based on univariable analysis showed that the proportion of patients with tremor at admission was not significantly higher in fatal cases $(20 \%)$ than in nonfatal cases $(3 \%)(20)$, but our multivariable analysis demonstrated that appearance of tremor at initial hospital visit was significantly associated with a fatal outcome.

Three patients had direct contact with the body fluid of ill companion animals ( 1 dog and 2 cats) before illness onset, and these patients reported no history of tick bite. Importantly, 2 of the 3 patients had direct contact with the saliva of an ill feral cat or an ill pet dog. In addition, the cat that bit patient no. 2 subsequently died (Table 3). These findings suggested that the ill companion animals could be the direct source of SFTSV infection.

Since 2017, a total of 24 pet cats living in western Japan have been diagnosed with SFTSV infection (30). All cats infected with SFTSV showed acute onset of clinical signs. High fever $\left(>39.5^{\circ} \mathrm{C}\right)$ was noted in $15 / 22$ cats $(68.2 \%)$ and vomiting in $10 / 24$ cats (41.7). Animal experiments by Park et al. (31) confirmed that cats are susceptible to SFTSV infection; the authors reported that $4 / 6$ cats infected with SFTSV died, and all cats showed signs that were similar to or more severe than those signs observed in humans infected with SFTSV. Those authors also found high viral loads in serum, saliva, and eye swabs taken 7 days postinfection from cats that subsequently died from infection. Collectively, these findings suggest that SFTSV transmission in patient no. 2 (Table 3) very possibly occurred from an ill cat through the patient's direct contact with the saliva of the cat.

Our study has several limitations. First, only 133/303 (43.9\%) patients reported to NESID were included in the study. Although we found no significant difference in sex and age distribution between the 133 patients who participated in this study and the 170 patients who did not, the 133 study participants might not represent the population of interest for research purposes. Second, although 3 patients 
who had contact with a body fluid of ill companion animals reported no history of a tick bite, whether these patients actually had a tick bite that was not reported because of recall bias is unknown. Therefore, we cannot exclude the possibility that the animals carried ticks and that a tick bite, rather than exposure to body fluid, might have been the actual mode of transmission. Third, no information is available on whether the 3 SFTS patients possibly infected from their ill companion animals were infected with the same strain of SFTSV as the animals. For any patients possibly infected from their companion animals, testing should be performed to determine whether the viruses isolated from the patient and from their animal are identical. In our study, we conducted the questionnaire surveys to evaluate the SFTSV infections from companion animals but were unable to determine the risk for SFTSV infection from these companion animals. Therefore, further case-control studies are required to determine the risk of exposure to the body fluids of companion animals.

In conclusion, we demonstrated that underlying malignant disease, low platelet count, and appearance of tremor at the first hospital visit were significantly associated with a fatal outcome among SFTS patients. The CFR of SFTS patients in Japan remained high at $27 \%$. Three $(2 \%)$ of 133 SFTS patients had direct contact with the body fluids of ill companion animals but no reported history of tick bite within the 2 weeks before illness onset, suggesting that ill companion animals might be a source of SFTSV transmission to humans. Although further studies on the epidemiologic and virologic analyses are needed, the owners of companion animals and veterinarians in the SFTS-endemic area should be fully aware of the risk of direct contact with body fluids of ill companion animals. The owners should avoid direct contact with body fluids, such as saliva, of their ill companion animals and should take care not to be bitten by them.

Members of the SFTS Epidemiological Research GroupJapan: Tsutomu Sakai (Noto General Hospital), Motoo Kobayashi (Municipal Tsuruga Hospital), Takeshi Ikeda (Ise Municipal General Hospital), Shigetoshi Sakabe (Japanese Red Cross Ise Hospital), Akihito Kitano, Hiroki Takatsu (Toyooka Public Hospital), Yutaka Shimazu, Kenichiro Kobayashi (Japanese Red Cross Wakayama Medical Center), Masako Kirishi, Yoshio Nakano (Kinan Hospital), Hiroki Hosoi (Kainan Iryou Center), Tomoaki Nagao (National Hospital Organization Minamiwakayama Medical Center), Fumina Matsuura, Mizuki Akiyoshi, Yuki Yamaguchi, Eisuke Okamoto (Japanese Red Cross Masuda Hospital), Ryosuke Ishida (Shimane Prefectural Central Hospital),
Shinya Watanabe (Okayama Kyoritsu General Hospital), Toshio Kuwai, Michiko Kida (National Hospital Organization Kure Medical Center), Mitsuaki Watanabe (Saiseikai Hiroshima Hospital), Norifumi Shigemoto (Hiroshima University Hospital), Yoshiro Hattori (Shobara Red Cross Hospital), Tsushima Hirofumi (Tsudhima Clinic), Wakako Harada, Eisuke Murakami (Hiroshima City Asa Citizens Hospital), Yuka Sano (Miyoshi Central Hospital), Tomohiro Taniguchi (Hiroshima Prefectural Hospital), Daisuke Tanioka (National Hospital Organization Iwakuni Clinical Center), Kaoru Yamamoto, Tsuru Masatoshi (National Hospital Organization Kanmon Medical Center), Shunsuke Hayashi, Munehiro Suzukawa, Aki Ishido (Shuto General Hospital), Tokio Matsunaga (Mine City Hospital), Toru Takahashi (Yamaguchi Prefectural Grand Medical Center), Naoki Fujita (Saiseikai Yamaguchi hospital), Takesuke Yamashita (Tokuyama Medical Association Hospital), Koji Yamashita (Tokuyama Central Hospital), Mari Otsuji (Yamaguchi University Hospital), Kazuhiko Ishizu (Tsushimi Hospital), Yoshinori Nakanishi, Osamu Hayabuchi (Tokushima Prefecture Naruto Hospital), Kengo Udaka (Tokushima Prefectural Central Hospital), Yoshinori Nakanishi (Tokushima Prefectural Kaifu Hospital), Tadashi Hayashi (Hayashi Clinic), Noriyasu Kondo, Takashi Hiraga (Katsuura Hospital), Tomoko Maruhashi (Tokushima University Hospital), Miho Saito (National Hospital Organization Tokushima Hospital), Hironobu Okumura (Clinic Okumura), Masato Waki (Kagawa Prefectural Central Hospital), Noriaki Miyake (Yashima General Hospital), Yoshito Homma, Chie Yamamoto (Ehime Prefectural Central Hospital), Kunihiko Oguro (Ehime Prefectural Minamiuwa Hospital), Masahiko Kaneko (Uwajima City Hospital), Yuichi Murakami (Kitaishikai Hospital), Tatsuya Konishi, Aiko Sakamoto (Matsuyama Red Cross Hospital), Koichiro Suemori (Ehime University Hospital), Makiko Oguro, Yukihide Yonekawa (Kihoku Municipal Kitauwa Hospital), Takashi Ogawa (Shimanto City Hospital), Risa Nakazawa, Shojiro Inada (Kochi Prefectural Hata Kenmin Hospital), Takanori Senba (Yawatahama City General Hospital), Shinsuke Kojima (Oonishi Hospital), Noriko Iwasaki (Saiseikai Fukuoka General Hospital), Takayuki Hoshina (Hospital of the University of Occupational and Environmental Health), Yohei Itoh (St.Mary's Hospital), Masaharu Miyahara, Aiko Nakamura (Karatsu Red Cross Hospital), Seijiro Makio (Saga University Hospital), Miki Yamauchi, Yuji Noguchi (Nagasaki Prefecture Tsushima Hospital), Yumi Yamamoto, Shinya Sato, Kazumasa Akagi (Nagasaki University Hospital), Hiroshi Takatani (Nagasaki Harbour Medical Center), Yasumori Izumi (National Hospital Nagasaki Medical Center), Keita Fujikawa (Isahaya General Hospital), Michiko Abe (Minamata City General Hospital and Medical Center), Masafumi Wada (Kamiamakusa City Hospital), Isao Sanada (Arao Municipal Hospital), Naohiro 
Taura (Hitoyoshi Medical Center), Kazuki Okuhiro (Oita Prefectural Hospital), Kokoro Honjyo (Oita University Hospital), Kunihiko Umekita (University of Miyazaki Hospital), Natsumi Uehara, Ryosuke Fujita (Miyazaki Prefectural Nobeoka Hospital), Atsushi Yamanaka, Takeshi Kawaguchi, Atsushi Naito, Hiroyuki Aratake (Miyazaki Prefectural Miyazaki Hospital), Masatoshi Tokojima (Saito Koyu Medical Center), Taku Miura (Kushima Municipal Hospital), Yasuhiko Araki (Nanbu Hospital), Bin Sakoda (Miyazaki Saiseikai Hyuga Hospital), Masahiro Kamekou (Kanoya Medical Center Citizens' Health Plaza), Yasuhiro Tanaka, Kouta Shigetou (National Hospital Organization Ibusuki Medical Center), Masaki Harada (Harada Clinic), Tetsushi Saihara (Soo Medical Association Hospital), Akihiko Arai (Idzuro Imamura Hospital), Shotaro Miyamoto (Kagoshima University Hospital), Hiroki Yamaguchi (Kagoshima Seikyo Hospital), Hirokazu Shimono (Sendai Medical Association Hospital), Haruhiko Ito (Yakushima Tokushyukai Hospital).

\section{Acknowledgments}

We thank the members of the SFTS Epidemiological Research Group-Japan and staff at local public health centers and public health institutes.

This study was supported by the Agency for Japan Medical Research and Development (grant no. 17fk0108202).

\section{About the Author}

Dr. Kobayashi is a clinical doctor and public health officer at Sayama Public Health Center and Medical Policy Division of Saitama Prefectural Government, Saitama, Japan, who has completed the field epidemiology training program at National Institute of Infectious Diseases, Tokyo, Japan. His research interests include infectious disease epidemiology and viral diseases such as severe fever with thrombocytopenia syndrome.

\section{References}

1. Yu XJ, Liang MF, Zhang SY, Liu Y, Li JD, Sun YL, et al. Fever with thrombocytopenia associated with a novel bunyavirus in China. N Engl J Med. 2011;364:1523-32. https:/ / doi.org/ 10.1056/NEJMoa1010095

2. Xu B, Liu L, Huang X, Ma H, Zhang Y, Du Y, et al. Metagenomic analysis of fever, thrombocytopenia and leukopenia syndrome (FTLS) in Henan Province, China: discovery of a new bunyavirus. PLoS Pathog. 2011; 7:e1002369. https://doi.org/10.1371/journal.ppat.1002369

3. Zhan J, Wang Q, Cheng J, Hu B, Li J, Zhan F, et al. Current status of severe fever with thrombocytopenia syndrome in China. Virol Sin. 2017;32:51-62. https:/ / doi.org/10.1007/ s12250-016-3931-1

4. Kim KH, Yi J, Kim G, Choi SJ, Jun KI, Kim NH, et al. Severe fever with thrombocytopenia syndrome, South Korea, 2012. Emerg Infect Dis. 2013;19:1892-4. https:/ / doi.org/10.3201/ eid1911.130792
5. Takahashi T, Maeda K, Suzuki T, Ishido A, Shigeoka T, Tominaga $\mathrm{T}$, et al. The first identification and retrospective study of severe fever with thrombocytopenia syndrome in Japan. J Infect Dis. 2014;209:816-27. https:/ / doi.org/ 10.1093/infdis/jit603

6. International Committee on Taxonomy of Viruses. Virus taxonomy: 2018b release. 2018 July [cited 2018 Jul 30]. https:/ / talk.ictvonline.org/taxonomy

7. Luo LM, Zhao L, Wen HL, Zhang ZT, Liu JW, Fang LZ, et al. Haemaphysalis longicornis ticks as reservoir and vector of severe fever with thrombocytopenia syndrome virus in China. Emerg Infect Dis. 2015;21:1770-6. https:/ / doi.org/ 10.3201/eid2110.150126

8. Yun SM, Lee WG, Ryou J, Yang SC, Park SW, Roh JY, et al. Severe fever with thrombocytopenia syndrome virus in ticks collected from humans, South Korea, 2013. Emerg Infect Dis. 2014;20:1358-61. https:/ / doi.org/10.3201/ eid2008.131857

9. Saijo M. Pathophysiology of severe fever with thrombocytopenia syndrome and development of specific antiviral therapy. J Infect Chemother. 2018;24:773-81. https:// doi.org/10.1016/j.jiac.2018.07.009

10. Tian H, Yu P, Chowell G, Li S, Wei J, Tian H, et al. Severe fever with thrombocytopenia syndrome virus in humans, domesticated animals, ticks, and mosquitoes, Shaanxi Province, China. Am J Trop Med Hyg. 2017;96:1346-9. https:// doi.org/10.4269/ajtmh.16-0333

11. Hwang J, Kang JG, Oh SS, Chae JB, Cho YK, Cho YS, et al. Molecular detection of severe fever with thrombocytopenia syndrome virus (SFTSV) in feral cats from Seoul, Korea. Ticks Tick Borne Dis. 2017;8:9-12. https://doi.org/10.1016/ j.ttbdis.2016.08.005

12. Liu Q, He B, Huang SY, Wei F, Zhu XQ. Severe fever with thrombocytopenia syndrome, an emerging tick-borne zoonosis. Lancet Infect Dis. 2014;14:763-72. https:/ / doi.org/ 10.1016/S1473-3099(14)70718-2

13. Tabara K, Fujita H, Hirata A, Hayasaka D. Investigation of severe fever with thrombocytopenia syndrome virus antibody among domestic bovines transported to slaughterhouse in Shimane Prefecture, Japan. Jpn J Infect Dis. 2016;69:445-7. https://doi.org/10.7883/yoken.JJID.2015.624

14. Lee SH, Kim HJ, Byun JW, Lee MJ, Kim NH, Kim DH, et al. Molecular detection and phylogenetic analysis of severe fever with thrombocytopenia syndrome virus in shelter dogs and cats in the Republic of Korea. Ticks Tick Borne Dis. 2017;8:626-30. https://doi.org/10.1016/j.ttbdis.2017.04.008

15. Niu G, Li J, Liang M, Jiang $X$, Jiang $M$, Yin $H$, et al. Severe fever with thrombocytopenia syndrome virus among domesticated animals, China. Emerg Infect Dis. 2013;19:75663. https://doi.org/10.3201/eid1905.120245

16. Bao CJ, Guo XL, Qi X, Hu JL, Zhou MH, Varma JK, et al. A family cluster of infections by a newly recognized bunyavirus in eastern China, 2007: further evidence of person-to-person transmission. Clin Infect Dis. 2011;53: 1208-14. https:// doi.org/10.1093/cid/cir732

17. Gai Z, Liang M, Zhang Y, Zhang S, Jin C, Wang SW, et al. Person-to-person transmission of severe fever with thrombocytopenia syndrome bunyavirus through blood contact. Clin Infect Dis. 2012;54:249-52. https:/ / doi.org/ 10.1093/cid/cir776

18. Kim WY, Choi W, Park SW, Wang EB, Lee WJ, Jee Y, et al. Nosocomial transmission of severe fever with thrombocytopenia syndrome in Korea. Clin Infect Dis. 2015;60:1681-3. https://doi.org/10.1093/cid/civ128

19. Saito T, Fukushima K, Umeki K, Nakajima K. Severe fever with thrombocytopenia syndrome in Japan and public health 
communication. Emerg Infect Dis. 2015;21:487-9.

https://doi.org/10.3201/eid2103.140831

20. Kato H, Yamagishi T, Shimada T, Matsui T, Shimojima M, Saijo M, et al.; SFTS Epidemiological Research Group-Japan. Epidemiological and clinical features of severe fever with thrombocytopenia syndrome in Japan, 2013-2014. PLoS One. 2016;11:e0165207. https://doi.org/10.1371/journal.pone. 0165207

21. Infectious Disease Surveillance Center, National Institute of Infectious Diseases. Infectious disease surveillance system in Japan. 2018 February 22 [cited 2018 Feb 23]. https:/ / www. niid.go.jp/niid/images/epi/nesid/nesid_en.pdf

22. Liu W, Lu QB, Cui N, Li H, Wang LY, Liu K, et al. Case-fatality ratio and effectiveness of ribavirin therapy among hospitalized patients in china who had severe fever with thrombocytopenia syndrome. Clin Infect Dis. 2013;57:1292-9. https:// doi.org/10.1093/cid/cit530

23. Chen Y, Jia B, Liu Y, Huang R, Chen J, Wu C. Risk factors associated with fatality of severe fever with thrombocytopenia syndrome: a meta-analysis. Oncotarget. 2017;8:89119-29. https://doi.org/10.18632/oncotarget.19163

24. Sun J, Gong Z, Ling F, Zhang R, Tong Z, Chang Y, et al. Factors associated with severe fever with thrombocytopenia syndrome infection and fatal outcome. Sci Rep. 2016;6:33175. https:/ / doi.org/10.1038/srep33175

25. Li H, Lu QB, Xing B, Zhang SF, Liu K, Du J, et al. Epidemiological and clinical features of laboratorydiagnosed severe fever with thrombocytopenia syndrome in China, 2011-17: a prospective observational study. Lancet Infect Dis. 2018;18:1127-37. https:/ / doi.org/10.1016/ S1473-3099(18)30293-7

26. Gai ZT, Zhang Y, Liang MF, Jin C, Zhang S, Zhu CB, et al. Clinical progress and risk factors for death in severe fever with thrombocytopenia syndrome patients. J Infect Dis. 2012;206:1095-102. https://doi.org/10.1093/infdis/jis472

27. Kida K, Matsuoka Y, Shimoda T, Matsuoka H, Yamada H, Saito T, et al. A case of cat-to-human transmission of severe fever with thrombocytopenia syndrome virus. Jpn J Infect Dis. 2019;72:356-8. https:// doi.org/10.7883/ yoken.JJID.2018.526

28. Yoshikawa T, Fukushi S, Tani H, Fukuma A, Taniguchi S, Toda S, et al. Sensitive and specific PCR systems for detection of both Chinese and Japanese severe fever with thrombocytopenia syndrome virus strains and prediction of patient survival based on viral load. J Clin Microbiol. 2014;52:3325-33. https://doi.org/10.1128/JCM.00742-14

29. Fukuma A, Fukushi S, Yoshikawa T, Tani H, Taniguchi S, Kurosu T, et al. Severe fever with thrombocytopenia syndrome virus antigen detection using monoclonal antibodies to the nucleocapsid protein. PLoS Negl Trop Dis. 2016;10:e0004595. https://doi.org/10.1371/journal. pntd.0004595

30. Matsuu A, Momoi Y, Nishiguchi A, Noguchi K, Yabuki M, Hamakubo E, et al. Natural severe fever with thrombocytopenia syndrome virus infection in domestic cats in Japan. Vet Microbiol. 2019;236:108346. https:/ / doi.org/ 10.1016/j.vetmic.2019.06.019

31. Park ES, Shimojima M, Nagata N, Ami Y, Yoshikawa T, Iwata-Yoshikawa N, et al. Severe fever with thrombocytopenia syndrome phlebovirus causes lethal viral hemorrhagic fever in cats. Sci Rep. 2019;9:11990. https://doi.org/10.1038/ s41598-019-48317-8

Address for correspondence: Kazunori Oishi, Toyama Institute of Health, 1-17 Nakataikouyama, Imizu, Toyama, 930-0363, Japan; email: toyamaeiken1@chic.ocn.ne.jp

\section{EID podcast Bird Migration and West Nile Virus in the U.S.}

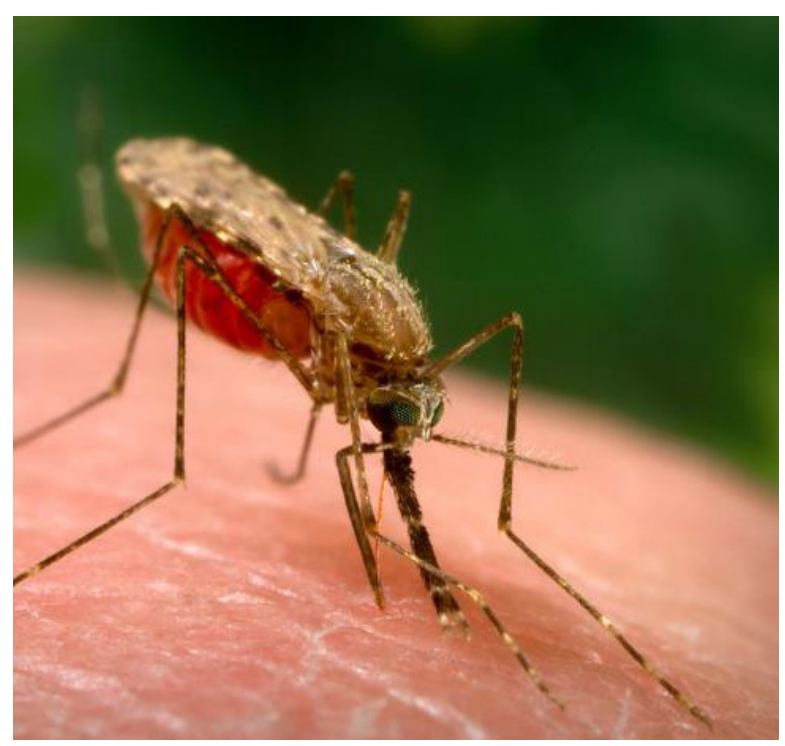

West Nile virus cycles between birds and mosquitoes that then infect humans through bites. But birds don't tend to stay in one place, and neither does the virus; a recent study has mapped how the disease has evolved along known migration routes.

In this EID podcast, Dr. Alan Barrett, Professor and Vice Chair for Research in the Department of Pathology at the University of Texas, Medical Branch, explores how bird migration patterns influence the epidemiology of West Nile virus in the U.S.

\section{Visit our website to listen: https: / /go.usa.gov/xy6n EMERGING INFECTIOUS DISEASES}

